\title{
Prosthodontic Rehabilitation of a Patient with Cleft Palate and Oronasal Fistula
}

\author{
${ }^{1}$ Rajani A Dable, ${ }^{2}$ Sheeba H Sharma, ${ }^{3}$ Kuldeep, ${ }^{4}$ Puneet Mutneja, ${ }^{5}$ Shyam U Jadhav
}

\begin{abstract}
Maxillofacial defects like oronasal fistulae present a functional problem due to the unsuccessful surgical reconstructive and repair attempts. The only option that remains with the patient is to go for maxillofacial obturation of the same. Prosthodontic rehabilitation of maxillary palatal defects in the anterior region is a crucial job for every prosthodontist as esthetics is of paramount importance here. Oronasal fistula results from congenital malformations or it can be an acquired defect resulting from surgery for oral neoplasms or trauma. The cleft palate patient is mainly characterized by the presence of an oronasal communication, malformation or agenesis of the teeth close to the cleft, and deficient sagittal and transverse growth of the maxilla. Prosthodontic rehabilitation depends on the conditions available, such as size and location of fistula, age of the patient, psychological status, and demand for esthetics. The present case report is about a patient with cleft lip and palate with an oronasal fistula (communication) that had recurred following the cleft reconstruction surgery. Out of various available prosthetic treatments, emphasis is being placed on the approach chosen to make it acceptable to the patient.
\end{abstract}

Keywords: Cleft lip, Modified Andrew's bridge, Oronasal communication, Oronasal fistula, Palate patient.

How to cite this article: Dable RA, Sharma SH, Kuldeep, Mutneja P, Jadhav SU. Prosthodontic Rehabilitation of a Patient with Cleft Palate and Oronasal Fistula. J Oral Health Comm Dent 2018;12(1):37-40.

Source of support: Nil

Conflict of interest: None

\section{INTRODUCTION}

The prevalence of cleft lip and palate among the general population depends on racial, ethnic, and geographic origin, as well as on socioeconomic status. It has been estimated as ranging from 1:500 to 1:2,500 live births. ${ }^{1,2}$ Cleft lip occurs in 20 to $30 \%$ of cases, cleft lip and palate in 35 to $50 \%$, and cleft palate alone in 30 to $45 \%$. $^{3}$

${ }^{1}$ Professor and Head, ${ }^{2}$ Professor, ${ }^{3,4}$ Reader, ${ }^{5}$ Postgraduate Student

${ }^{1-5}$ Department of Prosthodontics, Teerthanker Mahaveer Dental College \& Research Centre, Moradabad, Uttar Pradesh, India

Corresponding Author: Rajani A Dable, Professor and Head Department of Prosthodontics, Teerthanker Mahaveer Dental College \& Research Centre, Moradabad, Uttar Pradesh, India Phone: +919004949313, e-mail: rajnidable@gmail.com
The etiology of cleft lip and palate is complex and depends on genetic and environmental factors. ${ }^{1}$ Some authors $^{1-3}$ argue that the cleft palate is caused by an alteration in the normal fusion process. Other factors, such as a defect in vascular supply to the region involved; a mechanical alteration in tongue size; intoxication with substances, such as alcohol, drugs, or toxins; and infections or lack of development are also detected. In contrast, some authors attribute it to a serious defect produced by a mutant gene, or a small defect caused by several genes. ${ }^{3}$

The palatal defects can be either congenital or acquired. In India, the incidence of cleft lip and palate is 1 in 500 live births, and majority of these patients are not treated surgically at the appropriate time. ${ }^{4}$ Palatal fistula is a common complication arising out of cleft palate repair and its occurrence ranges from 0 to $34 \%$ as observed in various studies. $^{5}$

Prosthodontic care for the oronasal fistula patients becomes more critical when the obturation of defect and retention of the prosthesis demand meticulous execution. As the retention is the foremost factor in the successful prosthodontic treatment of cleft palate, precision attachments are used for the better results. They provide a twofold advantage to the patient, i.e., comfort through the fixed type and an easy maintenance through the removable type.

Oronasal fistula causes hypernasality due to escape of air during speech and also may lead to regurgitation of food and fluid to the nasal cavity. ${ }^{6}$ Various prosthetic treatment modalities are available to close the soft tissue defects, such as obturator, removable flange prosthesis, Andrew's bridge, and surgical bone augmentation. Fixed partial denture, such as the Andrew's bridge permits the rehabilitation of the defect with a fixed removable partial denture as an alternative method for conventional procedure. This prosthesis permits the replacement of the missing teeth as well as missing supporting structures necessary for proper function and esthetics. ${ }^{7}$ However, the increasing demand for esthetics in restorations can be met with any of the currently available ceramic restoration. In this case report, the rehabilitation of the oronasal defect is attempted with a fixed removable appliance using ceramic restoration for better esthetics. 


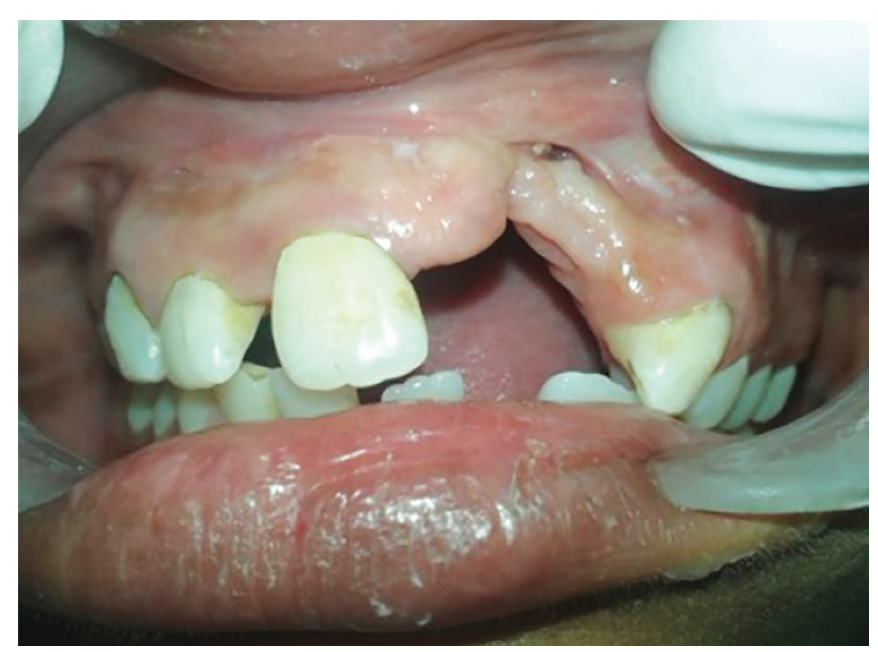

Fig. 1: Patient examination for the orofacial defect extensions

\section{CASE REPORT}

A 21-year-old female patient reported to the Department of Prosthodontics, with a chief complaint of missing front teeth. After examination, a postsurgery scar on left upper lip was noticed extraorally and an oronasal fistula intraorally (Fig. 1). A relevant history revealed that the patient underwent surgical correction at the age of 4 years, and due to surgical phobia, she rejected further reconstructive surgeries.

Intraoral examination revealed that the maxilla was having Veau's class III defect (unilateral cleft lip, alveolus, hard and soft palate) with missing left central and lateral incisor. A fistula measuring 3 to $4 \mathrm{~mm}$ in diameter was present in the labial part of the premaxilla (Fig. 1). The common complaints like regurgitation of food and nasal escape were absent except the unintelligible voice with a nasal twang.

Considering her age, an obturator attached to the Andrew's bridge (fixed removable) was planned. Intentional endodontic treatment was done with tooth \# 11, and tooth preparation for porcelain-fused-to-metal (PFM) crowns was completed for both the abutment teeth 11 and 23. Impression was recorded with rubber base material and was checked for the accuracy (Fig. 2). Wax copings were prepared on the abutment teeth, and the plastic coffee stirrer was adapted for fabrication of bar as per the anteroposterior and superoinferior availability of space to accommodate the artificial teeth and the retentive components (Fig. 3). The stirrer and the copings were cast together in $\mathrm{Ni}-\mathrm{Cr}-\mathrm{Co}$ alloy, and a finished and polished assembly was tried in mouth and checked for accuracy. After the undercut block out, the position of the riders and the smooth slide of the housing were checked (Fig. 4). Missing central and lateral incisors were planned to be replaced with PFM teeth rather than conventional acrylic teeth. A waxed-up denture base was prepared extending to the hard palate 8

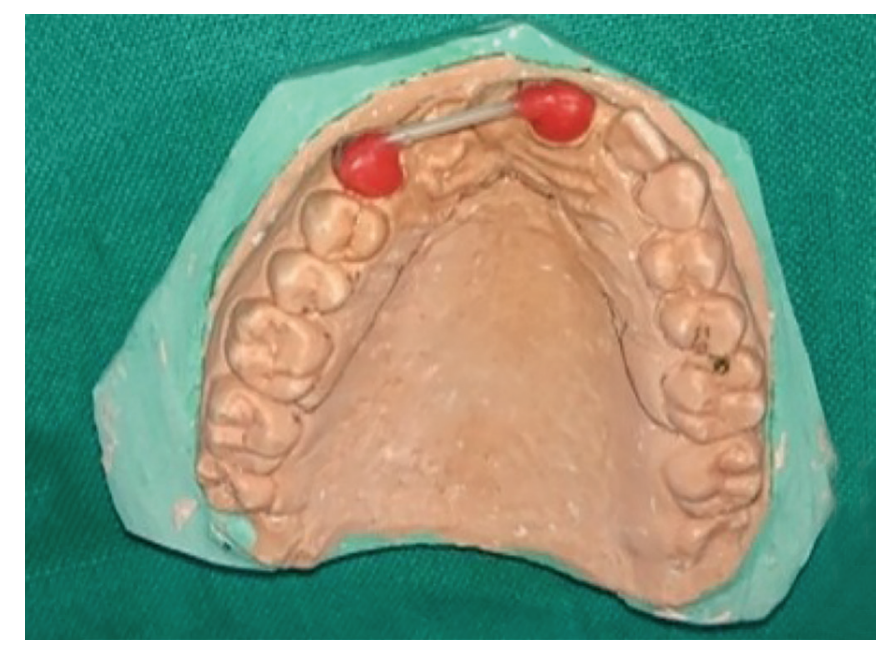

Fig. 2: Coffee straw cast attached to the wax pattern

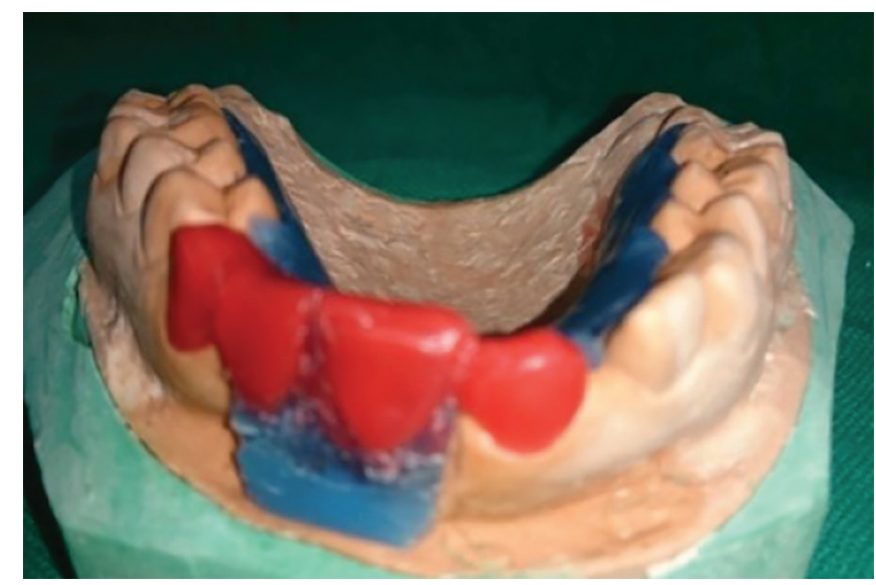

Fig. 3: Waxed-up fixed and removable dentures to be cast separately

to $10 \mathrm{~mm}$ palatally, the pontic patterns were attached to it and adjusted with the adjacent retainers. The crowns over the abutment teeth were cast separately along with the bar. The removable denture, along with the PFM pontics, was tried in patient's mouth, and checked for accuracy.

The replacement of teeth was accomplished in two ways: Fixed crowns were given on the abutment teeth, and the missing teeth were replaced by using similar material (PFM) but were attached to the removable plate that was seated on the bar and the riders (Fig. 5).

An acrylic plate was fabricated to obturate the buccal defect in conjunction with the removable metal denture base and was acrylized in heat-cured acrylic resin (Fig. 6). The nylon riders were positioned on the bar, and the metal housing was soldered to the intaglio surface of the denture base. The prosthesis was checked for fit and accuracy.

\section{DISCUSSION}

Oronasal fistula is a common complication of cleft palate surgical repair observed in different studies, and to correct 

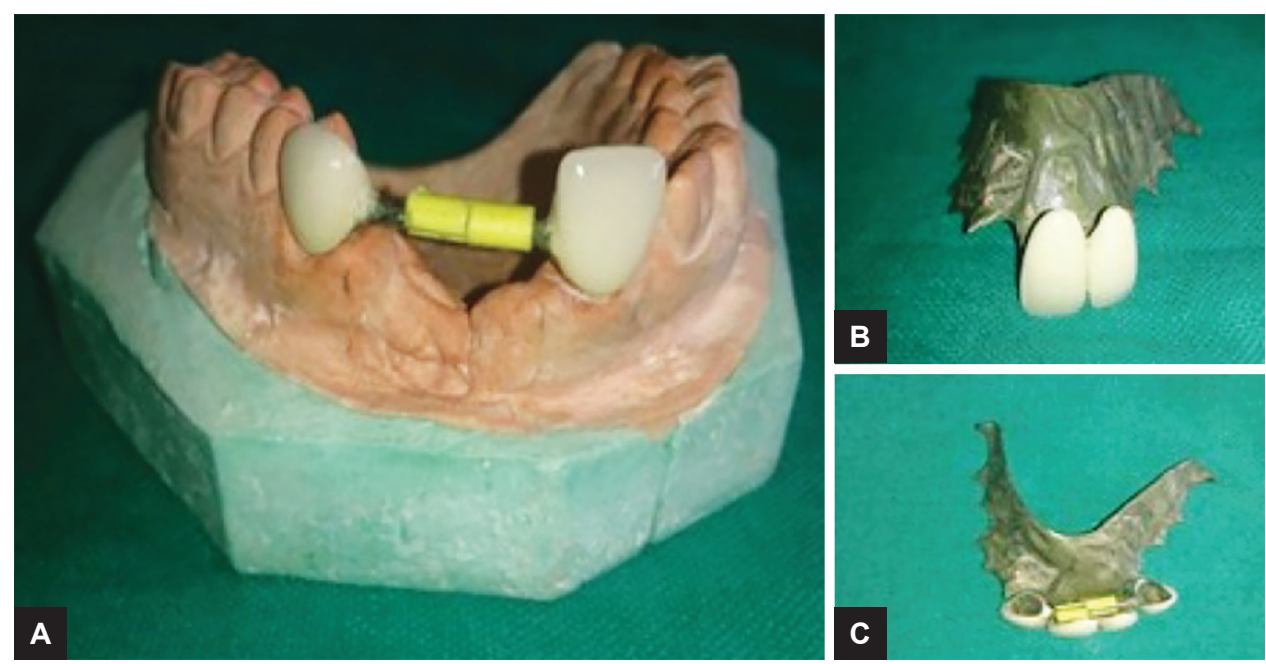

Figs 4A to C: $(A)$ Ceramic crowns over the abutment teeth; $(B)$ ceramic pontics attached to the removable plate; and $(\mathrm{C})$ removable and fixed prostheses together

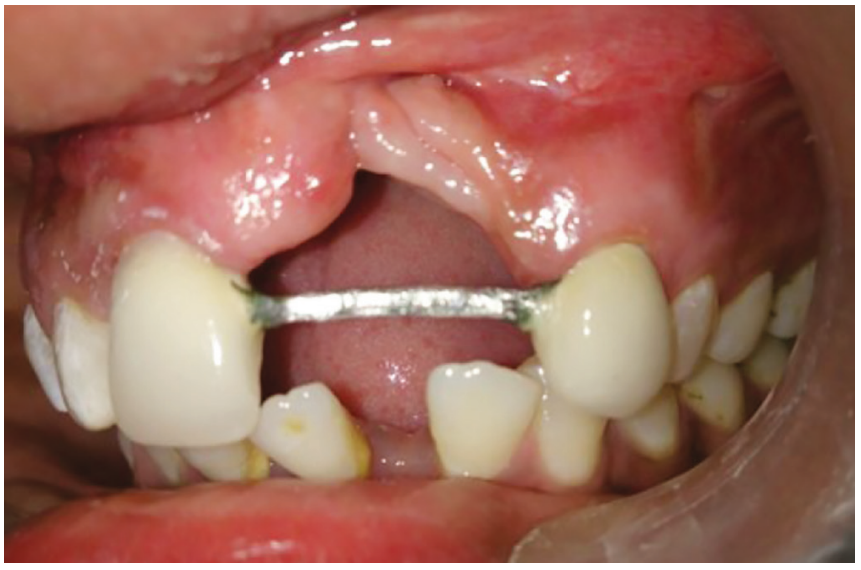

Fig. 5: Retainers in place

inevitable sequel of the surgery, the prosthetic rehabilitation with obturator is an auxiliary or complementary treatment to surgical treatments.

Attaching the obturator to the Andrew's bridge facilitates a more retentive prosthesis. Hence, the present case describes an unusual way of treatment modality where the Andrew's bridge was modified and attached to the removable denture base restoring the missing teeth that were fabricated by using ceramic material instead of acrylic, with the removable portion connected to the precision attachments.

Incorporation of precision attachments makes the removable portion of it to retain well, minimizing the need of extended denture base that ultimately improves the proprioception and taste perception, enhancing the patient comfort.

The treatment protocols in dentulous and edentulous patients differ, but the demand of better function and esthetics is always more in young patients. A similar case was managed by Harish et al by closing the defect with the help of an Andrew's bridge. ${ }^{8}$ The removable

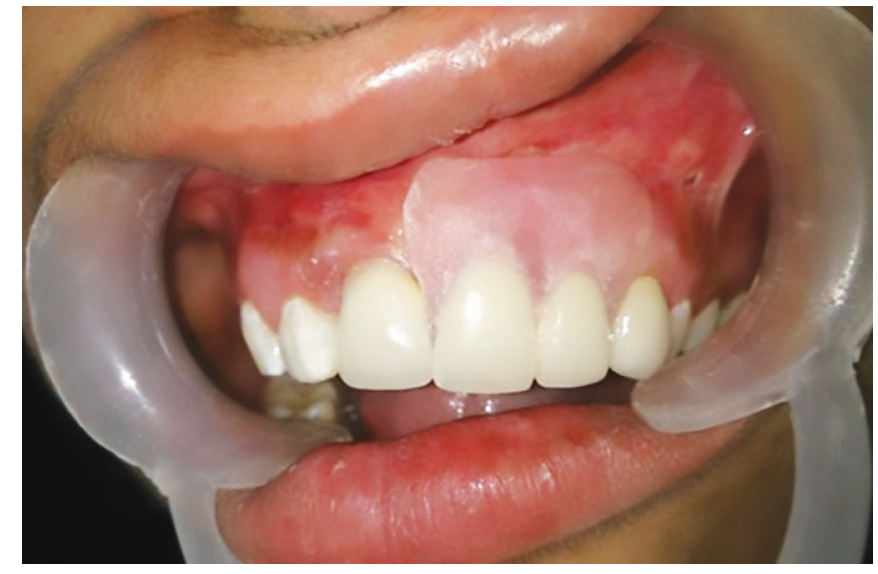

Fig. 6: Prosthesis with fixed and removable components in mouth

denture/obturator was fixed over the bar with the help of precision attachments. The known advantages here are enhanced stability and retention and also an effective defect obturation. Oronasal fistula poses a critical situation for a prosthodontist as it requires the restoration of function as well as the oronasal opening fulfilling the esthetic needs of the patient.

\section{CONCLUSION}

It is the primary right of every human being to look good and feel good. Cleft always makes the person conscious as it reduces the esthetic value of the face and disturbs the smile of a person. Palatal fistula is a common complication of a cleft palate surgical repair, and it is an inevitable sequel of the surgery. The prosthetic rehabilitation with obturator is an auxiliary and supportive treatment to surgical management.

\section{REFERENCES}

1. Slayton RL, Williams L, Murray JC, Wheeler JJ, Lidral AC, Nishimura CJ. Genetic association studies of cleft lip and/or 
palate with hypodontia outside the cleft region. Cleft Palate Craniofac J 2003 May;4(3):274-279.

2. Ohyama T. Prosthodontic considerations for patients with cleft lip and palate. Int Dent J 1986 Sep;36(3):140-145.

3. Abadi B, Johnson JD. The prosthodontic management of cleft palate patients. J Prosthet Dent 1982 Sep;48(3):297-302.

4. Ankola AV, Nagesh L, Hedge P, Karibasappa GN. Primary dentition status and treatment needs of children with cleft lip and/or palate. J Indian Soc Pedod Prev Dent 2005 Jun;23(2): 80-82.

5. Cohen SR, Kalinowski J, LaRossa D, Randall P. Cleft palate fistulas: a multivariate statistical analysis of prevalence, etiology, and surgical management. Plast Reconstr Surg 1991 Jun;87(6):1041-1047.

6. Inman DS, Thomas P, Hodgkinson PD, Reid CA. Oro-nasal fistula development and velopharyngeal insufficiency following primary cleft palate surgery-an audit of 148 children born between 1985 and 1997. Br J Plast Surg 2005 Dec;58(8): 1051-1054.

7. Immekus JE, Aramany M. A fixed removable partial denture for cleft palate patients. J Prosthet Dent 1975 Sep;34(3):286-291.

8. Harish PV, Bhojaraju N, Sowmya GR, Gangaiah M. Management of oro-nasal fistula using Andrew's bridge: a case report. J Indian Prosthodont Soc 2014 Sep;14(3):317-320. 